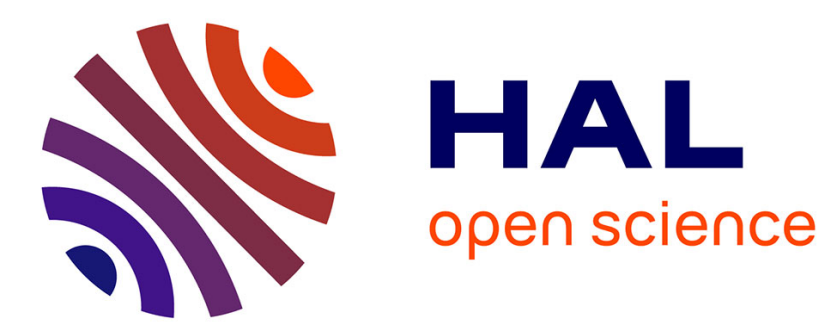

\title{
Vibration of a Surface-Coated Elastic Block Subject to Bending
}

\author{
M. Gei, R. W. Ogden
}

\section{To cite this version:}

M. Gei, R. W. Ogden. Vibration of a Surface-Coated Elastic Block Subject to Bending. Mathematics and Mechanics of Solids, 2002, 7 (6), pp.607-628. hal-01303471

\section{HAL Id: hal-01303471 \\ https://hal.science/hal-01303471}

Submitted on 18 Apr 2016

HAL is a multi-disciplinary open access archive for the deposit and dissemination of scientific research documents, whether they are published or not. The documents may come from teaching and research institutions in France or abroad, or from public or private research centers.
L'archive ouverte pluridisciplinaire HAL, est destinée au dépôt et à la diffusion de documents scientifiques de niveau recherche, publiés ou non, émanant des établissements d'enseignement et de recherche français ou étrangers, des laboratoires publics ou privés. 


\title{
Vibration of a Surface-Coated Elastic Block Subject to Bending
}

\author{
M. GEI \\ Dipartimento di Scienze e Metodi dell Ingegneria, Università di Modena e Reggio Emilia, Viale \\ Allegri 13, 1-42100 Reggio Emilia, Italy \\ R.W.OGDEN \\ Department of Mathematics, University of Glasgow, University Gandens, Glasgow GI2 $8 Q W, U K$
}

\begin{abstract}
In this paper we study the effect of a thin-film elastic surface coating on infinitesimal plane vibrations of a rectangular block of incompressible isotropic elastic material, which is subject to a static pure bending deformation. The equations goveming the vibrations superimposed on the bending deformation are derived along with appropriate boundary conditions. The equations are then solved numerically in order to illustrate the dependence of the frequency of vibrations on the aspect ratio and the underlying deformation of the block and on the material parameters of the coating. Comparisons with results for an uncoated block are highlighted and the limiting case of zero frequency (corresponding to bifurcation of the static configuration) is discussed.
\end{abstract}

Key Words: Nonlinear elasticity, vibrations, coating, bending

\section{INTRODUCTION}

In several recent papers, a static theory of an elastic surface coating for an elastic bulk material has been developed by Steigmann and Ogden [1,2,3], and several applications of the theory have been studied $[4,5]$. More recently, the dynamic counterpart of the theory has been presented [6]. The aim of this paper is to apply the dynamic theory to a prototype problem in order to illustrate the influence of the surface coating on the vibration characteristics of a rectangular block of incompressible isotropic elastic material subject to a finite static underlying deformation.

In section 2, following Ogden and Steigmann [6], we summarize the equations governing the plane strain dynamics of a thin-film surface coated solid subject to large deformations are summarized while in section 3 the corresponding incremental equations governing small motions superimposed on an underlying finite motion are given. The static deformation of a rectangular block into a sector of a circular cylindrical tube is described in section 4 as a prelude to considering a superimposed infinitesimal motion. Material models to be used for the bulk material and the surface coating are discussed in section 4.1. The incremental equations from section 3 are then specialized in section 5 for this underlying deformation 
and appropriate incremental boundary conditions are derived in a convenient form. This generalizes to the dynamic situation the work of Dryburgh and Ogden [5] concerned with the (static) bifurcation of a rectangular block subject to bending. In section 6 time-harmonic vibrations are considered and appropriate non-dimensionalizations for the various parameters are established. The resulting equations are solved numerically for a representative range of parameter values and the results are discussed in section 6.1. The frequency is plotted against a measure of the underlying static deformation, namely the stretch on the long curved side of the block. The results illustrate the effect of changing the bending stiffness of the coating and the aspect ratio of the block, and a comparison is made with corresponding results for an uncoated block. The dependence of the frequency on the ratio of coating to bulk density and the relative importance of the coating rotatory inertia term are also discussed briefly.

\section{DYNAMICS OF A SURFACE-COATED BODY}

We consider the plane motion of a body whose plane section occupies the region $\mathcal{C} \in \mathbb{R}^{2}$ in the reference (natural) configuration. The current configuration, which changes with time $t$, is denoted by $\hat{\mathcal{C}}$ and the motion is described by the invertible and appropriately regular function

$$
\chi: \mathcal{C} \times \mathcal{I} \rightarrow \hat{\mathcal{C}},(\mathbf{X}, t) \rightarrow \mathbf{x}=\chi(\mathbf{X}, t)
$$

where $\mathbf{X}$ and $\mathbf{x}$ are points of $\mathcal{C}$ and $\hat{\mathcal{C}}$, respectively, and $\mathcal{I}$ is a time interval. The deformation gradient $\mathbf{F}(\mathbf{X}, t)$ is given by $\mathbf{F}=\operatorname{Grad} \boldsymbol{\chi}$, where Grad is the two-dimensional gradient operator on $\mathcal{C}$.

Let the boundary $\partial \mathcal{C}$ be parametrized by arclength $S$, taken in the counterclockwise sense such that the outward unit normal $\mathbf{N}$ is rightward following the orientation of the curve. Similarly, points of $\partial \hat{\mathcal{C}}$ are identified by the arclength $s$ and the outward unit normal on $\partial \hat{\mathcal{C}}$ is denoted by $\mathbf{n}$, which is related to $\mathbf{N}$ by the classical Nanson's formula, which, for the present plane-strain specialization, has the form

$$
\mathbf{n} \mathrm{d} s=\mathbf{F}^{-T} \mathbf{N} \mathrm{d} S .
$$

Let part of the boundary $P \subset \partial \mathcal{C}$ be coated with a thin elastic film that deforms as a material curve to become $\hat{P} \subset \partial \hat{\mathcal{C}}$. The motion of each point $\mathbf{X}(S) \in P$ is described by the position vector

$$
\mathbf{r}(S, t)=\chi(\mathbf{X}(S), t)
$$
to

In terms of the notation defined by ()$^{\prime}=\partial() / \partial S$, the unit tangent $\mathbf{T}=\mathbf{X}^{\prime}(S)$ to $P$ maps

$$
\mathbf{r}^{\prime}(S, t)=\mathbf{F}(\mathbf{X}(S), t) \mathbf{T}(S)=\lambda(S, t) \boldsymbol{\tau}(S, t)
$$


where $\lambda(S, t)=|\mathbf{F}(\mathbf{X}(S), t) \mathbf{T}(S)|=s^{\prime}(S, t)$ is the stretch of $P$ induced by the motion and $\boldsymbol{\tau}(S, t)$ is the unit tangent to $\hat{P}$ at the point corresponding to arclength $S$ on $P$.

The unit tangent $\tau$ can be decomposed into the directions singled out by a Cartesian basis $\left\{\mathbf{e}_{1}, \mathbf{e}_{2}\right\}$ through the counterclockwise angle $\varphi(S, t)$ between its direction and that of $\mathbf{e}_{1}$. Thus

$$
\boldsymbol{\tau}(S, t)=\cos \varphi(S, t) \mathbf{e}_{1}+\sin \varphi(S, t) \mathbf{e}_{2} .
$$

We denote by $\boldsymbol{\nu}(S, t)=\mathbf{k} \times \boldsymbol{\tau}(S, t)$ the leftward unit normal to $\hat{P}$, where $\mathbf{k}=\mathbf{e}_{1} \times \mathbf{e}_{2}$. Differentiation of Equation (5) with respect to $S$ yields

$$
\boldsymbol{\tau}^{\prime}(S, t)=\kappa(S, t) \boldsymbol{\nu}(S, t), \quad \kappa(S, t)=\varphi^{\prime}(S, t),
$$

and we note that the curvature of $\hat{P}$ is $\lambda^{-1} \kappa$.

The material in $\mathcal{C}$ is assumed to be hyperelastic, with strain-energy function $W(\mathbf{F})$. It is also assumed to be incompressible, so that the constraint $\operatorname{det} \mathbf{F}=1$ is satisfied. The nominal stress tensor $\mathbf{S}$ is then given by

$$
\mathbf{S}=\frac{\partial W}{\partial \mathbf{F}}-p \mathbf{F}^{-1}
$$

where $p$ is the usual Lagrange multiplier.

The surface coating is taken to be characterized by the strain energy $U(\lambda, \kappa)$ per unit length [1] which accounts for elastic resistance to both extension and flexure of $P$. The tangential force $F$ and moment $M$ on $\hat{P}$ are given respectively by

$$
F=U_{, \lambda}, \quad M=U_{, \kappa},
$$

where a comma indicates a partial derivative.

In general, the force, denoted $\mathbf{f}$, at a point on $\hat{P}$ has tangential component equal to $F$ and also a normal component, $G$ say, so that we can write

$$
\mathbf{f}=F \boldsymbol{\tau}+G \boldsymbol{\nu}
$$

The quantity $G$ may be interpreted as the transverse shear force on the film. It is a Lagrange multiplier associated with the constraint that no transverse shear strain is admitted because of the assumed geometrical nature of the coating. It is not determined by a constitutive equation and is therefore an unknown of the problem requiring a specific equation for its determination.

In the absence of body forces the equations of motion in $\mathcal{C}$ take the form

$$
\operatorname{Div} \mathbf{S}=\rho \mathrm{D}^{2} \chi / \mathrm{D} t^{2}
$$

where $\rho$ is the mass density of the bulk material per unit area of $\mathcal{C}$, Div is the divergence operator in $\mathcal{C}$ and $\mathrm{D} / \mathrm{D} t$ denotes the material time derivative.

If we denote by $\partial \mathcal{C}_{x}$ and $\partial \mathcal{C}_{f}$ parts of $\partial \mathcal{C}$ without coating on which, respectively, displacements and tractions are prescribed, the appropriate boundary conditions are 


$$
\begin{gathered}
\mathbf{x}=\boldsymbol{\xi}(t) \quad \text { on } \partial \mathcal{C}_{x}, \\
\mathbf{S}^{T} \mathbf{N}=\mathbf{t}(t) \text { on } \partial \mathcal{C}_{f} .
\end{gathered}
$$

Further boundary conditions are provided by derivation of the equations governing the motion of the surface coating. Linear momentum and moment-of-momentum balances for the film give the following local equations [6]

$$
\begin{gathered}
\mathbf{f}^{\prime}=\mathbf{S}^{T} \mathbf{N}+\rho_{0} \mathrm{D}^{2} \mathbf{r} / \mathrm{D} t^{2} \text { on } P, \\
M^{\prime}+\lambda G=I \mathrm{D}^{2} \varphi / \mathrm{D} t^{2} \text { on } P,
\end{gathered}
$$

where $\rho_{0}$ and $I(S)$, respectively, are the mass density and the mass moment of inertia per unit reference length of the film, the latter taken to be independent of the motion.

Equation (13) couples the response of the bulk material with that of the film, while (14) determines the Lagrange multiplier $G(S, t)$, so that the force $\mathbf{f}(S, t)$ at each point of the coating is fully determined. In deriving (14) the rotatory inertia term has been taken into account. The importance of this term will be emphasized in the application considered in sections 5 and 6, and situations where its neglect can be justified will be identified. We refer to [6] for a detailed discussion of this point.

\section{INCREMENTAL MOTIONS}

We now consider an incremental motion superimposed on the dynamic deformation described in section 2. Incremental displacements are given by $\mathbf{u}(\mathbf{x}, t)=\dot{\mathbf{x}}(\mathbf{X}, t)$, where $(\dot{)})$ denotes a small increment in the quantity concerned. The incremental versions of the equations of motion referred to the reference and current configurations, respectively, are

$$
\operatorname{Div} \dot{\mathbf{S}}=\rho \mathrm{D}^{2} \dot{\mathbf{x}} / \mathrm{D} t^{2} \quad \text { in } \mathcal{C}
$$

and

$$
\operatorname{div} \boldsymbol{\Sigma}=\rho \mathrm{D}^{2} \mathbf{u} / \mathrm{D} t^{2} \text { in } \hat{\mathcal{C}}
$$

where div is the divergence operator in $\hat{\mathcal{C}}, \boldsymbol{\Sigma}=\mathbf{F} \dot{\mathbf{S}}$. We note that because of the incompressibility constraint the density $\rho$ of the bulk material is unaffected by the change of reference configuration.

The definition of the tensor $\boldsymbol{\Sigma}$ originates from the equality $\dot{\mathbf{S}}^{T} \mathbf{N}=\lambda \boldsymbol{\Sigma}^{T} \mathbf{n}$, which expresses the incremental traction vector in terms of the two incremental stress tensors, and the two-dimensional specialization $\lambda \mathbf{n}=\mathbf{F}^{-T} \mathbf{N}$ of Nanson's formula, obtained from (2) with $\lambda=\mathrm{d} s / \mathrm{d} S$. The linearized constitutive equation for the bulk material is

$$
\boldsymbol{\Sigma}=\mathbb{C}_{0} \boldsymbol{\Gamma}-\dot{p} \mathbf{I}
$$


where $\boldsymbol{\Gamma}=\operatorname{grad} \mathbf{u}$ (grad being the gradient operator in $\hat{\mathcal{C}}), \mathbf{I}$ is the two-dimensional identity tensor and $\mathbb{C}_{0}$ is the fourth-order tensor of instantaneous elastic moduli (possessing the major symmetry $C_{0 i j k l}=C_{0 k l i j}, i, j, k, l \in\{1,2\}$ ). Incompressibility requires that $\operatorname{tr} \boldsymbol{\Gamma}=0$.

Let $\boldsymbol{\sigma}$ denote the Cauchy stress tensor and $\sigma_{1}$ and $\sigma_{2}$ its principal components. Since $\boldsymbol{\Sigma}=\dot{\boldsymbol{\sigma}}-\boldsymbol{\Gamma} \boldsymbol{\sigma}$, the balance of rotational momentum yields $\Sigma_{12}-\Sigma_{21}=\sigma_{1} \Gamma_{21}-\sigma_{2} \Gamma_{12}$, and a comparison of this with (17) shows that

$$
C_{0 i j i j}=C_{0 j i i j}+\sigma_{i}, \quad i, j \in\{1,2\}, i \neq j .
$$

Specific expressions for $C_{0 i j k l}$ are given in section 5, where isotropic materials will be examined.

The incremental boundary conditions for the field equation (16) can be derived from (11)-(14). In particular, for points of $\partial \hat{\mathcal{C}}$ where there is no coating, we have

$$
\begin{gathered}
\mathbf{u}=\dot{\boldsymbol{\xi}}(t) \quad \text { on } \partial \hat{\mathcal{C}}_{x}, \\
\boldsymbol{\Sigma}^{T} \mathbf{n}=\dot{\mathbf{t}}(t) \quad \text { on } \partial \hat{\mathcal{C}}_{f} .
\end{gathered}
$$

On $\hat{P}$, by taking the increments of (13) and (14), updating from the variable $S$ to $s$, and noting that $\lambda=\mathrm{d} s / \mathrm{d} S$, we obtain

$$
\begin{gathered}
\dot{\mathbf{f}}^{\prime}(s, t)=\boldsymbol{\Sigma}^{T} \mathbf{n}+\lambda^{-1} \rho_{0} \mathrm{D}^{2} \mathbf{w}(s, t) / \mathrm{D} t^{2} \text { on } \hat{P}, \\
\dot{M}^{\prime}(s, t)+\lambda^{-1} \dot{\lambda} G+\dot{G}=\lambda^{-1} I \mathrm{D}^{2} \dot{\varphi}(s, t) / \mathrm{D} t^{2} \text { on } \hat{P},
\end{gathered}
$$

where ()$^{\prime}$ now indicates differentiation with respect to $s$ and $\mathbf{w}(\mathbf{x}, t)=\mathbf{u}(\mathbf{x}, t)$ for $\mathbf{x} \in \hat{P}$.

As pointed out in [6], if the underlying deformation is purely static then the form of the inertia term in Equation (22) is correct whether or not $I$ is strain dependent because the equations are linearized with respect to a fixed configuration of the film. Note also that $\lambda^{-1} \rho_{0}$ and $\lambda^{-1} I$, respectively, are the mass density and mass moment of inertia of the film per unit current length. Just as (14) determines $G$, Equation (22) essentially provides an expression for $\dot{G}$.

Since the incremental form of (9) may be written $\dot{\mathbf{f}}=\dot{F} \boldsymbol{\tau}+\dot{G} \boldsymbol{\nu}+F \dot{\boldsymbol{\tau}}+G \dot{\boldsymbol{\nu}}$, use of the expressions $\dot{\boldsymbol{\tau}}=\dot{\varphi} \boldsymbol{\nu}$ and $\dot{\boldsymbol{\nu}}=-\dot{\varphi} \boldsymbol{\tau}$ yields

$$
\dot{\mathbf{f}}=(\dot{F}-G \dot{\varphi}) \boldsymbol{\tau}+(F \dot{\varphi}+\dot{G}) \boldsymbol{\nu}
$$

The term $\dot{G}$ is given by (22), while $G$ can be obtained from the updated counterpart of (14), namely

$$
M^{\prime}(s, t)+G(s, t)=\lambda^{-1} I \mathrm{D}^{2} \varphi(s, t) / \mathrm{D} t^{2} .
$$

Furthermore, use of $\boldsymbol{\tau}^{\prime}=\varphi^{\prime} \boldsymbol{\nu}=\lambda^{-1} \kappa \boldsymbol{\nu}, \boldsymbol{\nu}^{\prime}=-\varphi^{\prime} \boldsymbol{\tau}=-\lambda^{-1} \kappa \boldsymbol{\tau}$ and $\dot{\varphi}^{\prime}=\lambda^{-1} \dot{\kappa}$ enables us to obtain an expression for $\mathbf{f}^{\prime}$, which is needed in (21). Thus

$$
\dot{\mathbf{f}}^{\prime}=T \boldsymbol{\tau}+N \boldsymbol{\nu} \text { on } \hat{P}
$$


where

$$
\begin{aligned}
T= & \dot{F}^{\prime}+\lambda^{-1} \dot{\kappa} M^{\prime}+M^{\prime \prime} \dot{\varphi}-\lambda^{-1} \kappa\left(F \dot{\varphi}+M^{\prime} \lambda^{-1} \dot{\lambda}-\dot{M}^{\prime}+\lambda^{-1} I \mathrm{D}^{2} \dot{\varphi} / \mathrm{D} t^{2}\right) \\
& +\lambda^{-1} I \mathrm{D}^{2} \varphi / \mathrm{D} t^{2}\left(\lambda^{-1} \lambda^{\prime} \dot{\varphi}+\lambda^{-2} \dot{\lambda} \kappa-\lambda^{-1} \dot{\kappa}\right)-\lambda^{-1} I \dot{\varphi} \mathrm{D}^{2} \varphi^{\prime} / \mathrm{D} t^{2} \\
N= & \lambda^{-1} \dot{\kappa} F+F^{\prime} \dot{\varphi}+\lambda^{-1} M^{\prime}\left(\dot{\lambda}^{\prime}-\lambda^{-1} \lambda^{\prime} \dot{\lambda}\right)+\lambda^{-1} \dot{\lambda} M^{\prime \prime}-\dot{M}^{\prime \prime} \\
& +\lambda^{-1} \kappa\left(\dot{F}+M^{\prime} \dot{\varphi}\right)+\lambda^{-1} I\left(\mathrm{D}^{2} \dot{\varphi}^{\prime} / \mathrm{D} t^{2}-\lambda^{-1} \lambda^{\prime} \mathrm{D}^{2} \dot{\varphi} / \mathrm{D} t^{2}\right) \\
& +\lambda^{-2} I \mathrm{D}^{2} \varphi / \mathrm{D} t^{2}\left(2 \lambda^{-1} \lambda^{\prime} \dot{\lambda}-\dot{\lambda}^{\prime}-\kappa \dot{\varphi}\right)-\lambda^{-2} \dot{\lambda} I \mathrm{D}^{2} \varphi^{\prime} / \mathrm{D} t^{2} .
\end{aligned}
$$

For future reference we record the incremental kinematical expressions

$$
\dot{\lambda}=\lambda \tau \cdot \mathbf{w}^{\prime}, \quad \dot{\kappa}=\lambda \nu \cdot \mathbf{w}^{\prime \prime}-\kappa \tau \cdot \mathbf{w}^{\prime}, \quad \dot{\varphi}=\nu \cdot \mathbf{w}^{\prime}
$$

which follow directly from (4) and (6).

\section{BENDING OF A SURFACE-COATED ELASTIC BLOCK}

In the application considered in sections 5 and 6 the effect of an incremental motion superimposed on the quasi-static finite deformation of a coated rectangular isotropic elastic block under flexure will be examined, so that time dependence in the equations that govern the finite motion is omitted. Here, we summarize the equations describing this type of inhomogeneous deformation are summarized.

In a Cartesian coordinate system $\left(X_{1}, X_{2}\right)$ the undeformed block $\mathcal{C}$ occupies the region

$$
-A \leq X_{1} \leq A, \quad-H \leq X_{2} \leq H
$$

The bending deformation is described, in terms of polar coordinates $(r, \theta)$, by

$$
r=f\left(X_{1}\right), \quad \theta=g\left(X_{2}\right),
$$

so that the deformed block $\hat{\mathcal{C}}$ is defined by

$$
f(-A) \leq r \leq f(A), \quad g(-H) \leq \theta \leq g(H) .
$$

Imposition of the incompressibility constraint reduces the deformation to

$$
r=\left(\frac{2 X_{1}}{\alpha}+\beta\right)^{1 / 2}, \quad \theta=\alpha X_{2},
$$

where $\alpha$ and $\beta$ are constants which are fixed by the boundary conditions (see, for example, Ogden [7]).

With respect to a reference rectangular Cartesian basis $\left\{\mathbf{e}_{1}, \mathbf{e}_{2}\right\}$ and to a current polar basis $\left\{\mathbf{e}_{r}, \mathbf{e}_{\theta}\right\}$, the (in-plane) deformation gradient takes the form 


$$
\mathbf{F}=\lambda^{-1} \mathbf{e}_{r} \otimes \mathbf{e}_{1}+\lambda \mathbf{e}_{\theta} \otimes \mathbf{e}_{2},
$$

where $\lambda_{r}=\lambda^{-1}$ and $\lambda_{\theta}=\lambda=\alpha r$ are the principal stretches associated with the Eulerian principal directions $\mathbf{e}_{r}$ and $\mathbf{e}_{\theta}$, respectively.

For an isotropic elastic material the strain-energy function $W(\mathbf{F})$ can be written, in the present plane context, as a function of the principal stretches, namely $W\left(\lambda_{r}, \lambda_{\theta}\right)$. Hence, through (7), the nominal stress tensor may be written

$$
\mathbf{S}=t_{1} \mathbf{e}_{1} \otimes \mathbf{e}_{r}+t_{2} \mathbf{e}_{2} \otimes \mathbf{e}_{\theta}
$$

where

$$
t_{1}=\frac{\partial W}{\partial \lambda_{r}}-p \lambda_{r}^{-1}, \quad t_{2}=\frac{\partial W}{\partial \lambda_{\theta}}-p \lambda_{\theta}^{-1} .
$$

The equilibrium equations obtained from (10) reduce to the following single ordinary differential equation in $X_{1}$ (see [7] for details)

$$
\frac{\mathrm{d} t_{1}}{\mathrm{~d} X_{1}}=\alpha t_{2}
$$

the integration of which, with the help of (34), yields

$$
t_{1}=(\tilde{W}+\eta) \lambda .
$$

In (36) the integration constant $\eta$ and the notation $\tilde{W}(\lambda)=W\left(\lambda^{-1}, \lambda\right)$ have been introduced. In terms of $\tilde{W}$, the difference between the Cauchy principal stresses $\sigma_{2}=\sigma_{\theta}=\lambda t_{2}$ and $\sigma_{1}=\sigma_{r}=\lambda^{-1} t_{1}$ is

$$
\sigma_{\theta}-\sigma_{r}=\lambda \frac{\partial \tilde{W}}{\partial \lambda}
$$

On the curved boundaries of the deformed body the traction vector is given by

$$
\mathbf{S}^{T} \mathbf{N}= \pm t_{1} \mathbf{e}_{r} \quad \text { on } X_{1}= \pm A,
$$

while on the straight sides $\theta= \pm \alpha H$ the resultant (in modulus) of the normal stress $t_{2}$, denoted $\mathcal{L}$, and its moment calculated about the origin, denoted $\mathcal{M}$, are

$$
\mathcal{L}=\int_{-A}^{A} t_{2} \mathrm{~d} X_{1}=\frac{1}{\alpha}\left[t_{1}\right]_{-A}^{A}, \quad \mathcal{M}=\int_{-A}^{A} r t_{2} \mathrm{~d} X_{1} .
$$

The boundaries $X_{1}=\mp A$, denoted $P^{-}$and $P^{+}$(with $P=P^{-} \cup P^{+}$), are coated with an elastic thin film with strain energy $U$ per unit length. Parametrization of $P$ is set so that $S=H-X_{2}$ on $P^{-}$and $S=H+X_{2}$ on $P^{+}$. Therefore, since $\varphi=\theta+3 \pi / 2$ on $P^{-}$and $\varphi=\theta+\pi / 2$ on $P^{+}$, the kinematical quantities introduced previously on the two coated sides of the block are 


$$
\left.\left.\begin{array}{l}
\lambda_{-}=\lambda(-A)=\alpha r_{-} \\
\kappa_{-}=-\theta^{\prime}\left(X_{2}\right)=-\alpha \\
\boldsymbol{\nu}=\mathbf{e}_{r}=-\mathbf{n} \\
\boldsymbol{\tau}=-\mathbf{e}_{\theta}
\end{array}\right\} \text { on } P^{-}, \begin{array}{l}
\lambda_{+}=\lambda(A)=\alpha r_{+} \\
\kappa_{+}=\theta^{\prime}\left(X_{2}\right)=\alpha \\
\boldsymbol{\nu}=-\mathbf{e}_{r}=-\mathbf{n} \\
\boldsymbol{\tau}=\mathbf{e}_{\theta}
\end{array}\right\} \text { on } P^{+}
$$

where subscripts - and + refer to $P^{-}$and $P^{+}$respectively.

Along each coated boundary, $\lambda$ and $\kappa$ are constant and, since the coating material is homogeneous, it follows that $F^{\prime}=M^{\prime}=0$ and, from (14) specialized to the static case, that $G=0$. Consequently, $\mathbf{f}(S)=U_{, \lambda} \boldsymbol{\tau}$ and $\mathbf{f}^{\prime}(S)=\kappa U_{, \lambda} \boldsymbol{\nu}$ on $P^{-}$and $P^{+}$. From (13), (36) and (38) it then follows that the boundary conditions that couple the response of the block to that of the elastic coating are

$$
t_{1}=(\tilde{W}+\eta) \lambda=-\kappa U_{, \lambda} \quad \text { on } P^{-} \text {and } P^{+} .
$$

These conditions, together with (39), provide sufficient information to determine $\alpha, \beta, \eta, \mathcal{L}$ and $\mathcal{M}$. For an uncoated block, whatever the strain-energy function $\tilde{W}(\lambda)$, the condition $\lambda_{-} \lambda_{+}=1$ holds, leading to

$$
\beta=\frac{\sqrt{1+4 A^{2} \alpha^{2}}}{\alpha}, \quad \mathcal{L}=0 .
$$

Steigmann and Ogden [2] have shown that a necessary condition for a static deformed configuration $(\lambda, \kappa)$ to be an energy minimizer is that

$$
\text { the Hessian matrix }\left[\begin{array}{cc}
U_{, \lambda \lambda} & U_{, \lambda \kappa} \\
U_{, \lambda \kappa} & U_{, \kappa \kappa}
\end{array}\right] \text { is positive semi-definite. }
$$

For a membrane film, for which bending stiffness is disregarded, a necessary condition is that $F$ is non-compressive, and hence

$$
U_{, \lambda} \geq 0
$$

\subsection{Material models}

We assume that the bulk material is characterized by a two-dimensional form of the general class of strain-energy functions introduced in [8]. Thus, we may write

$$
\tilde{W}(\lambda)=\sum_{i=1}^{N} \frac{\mu_{i}}{\delta_{i}}\left(\lambda^{\delta_{i}}+\lambda^{-\delta_{i}}-2\right),
$$

where $\mu_{i}$ and $\delta_{i}$ are constant parameters satisfying $\mu_{j} \delta_{j}>0$ (no summation over index $j$ ) and the ground-state shear modulus is $\mu=\sum_{i=1}^{N} \mu_{i} \delta_{i} / 2$.

In particular, a three-term form of (45) is used in the results shown in section 6.1, with values of the parameters given by

$$
\begin{aligned}
& \delta_{1}=1.3, \quad \delta_{2}=5.0, \quad \delta_{3}=-2.0, \\
& \mu_{1}=1.491 \mu, \quad \mu_{2}=0.003 \mu, \quad \mu_{3}=-0.0237 \mu \text {. }
\end{aligned}
$$


For the coating material we take $U$ to have the simple form

$$
U(\lambda, \kappa)=\frac{1}{2} m(\lambda-1)^{2}+\frac{1}{2} n \kappa^{2},
$$

where the material constants $m$ and $n$, for consistency with the requirement discussed in (43), are non-negative. The current axial force and couple in the coating ( $\mathrm{cf}(8))$ are given by

$$
F=m(\lambda-1), \quad M=n \kappa .
$$

As discussed in [1], from a comparison with engineering plate theory it can be deduced that the quantity $\sqrt{n / m}$, which has dimensions of length, may be interpreted as being of the order of magnitude of the coating thickness. Therefore, since the theory of surface coating applies to problems where the coating thickness is very small compared with any other length scale we shall restrict our attention to values of $m$ and $n$ such that $n / m \ll A^{2}$.

Having defined the strain-energy functions for the block and the coating, it is now possible to find the parameters that characterize the finite deformation of the coated block. In [5] explicit results for a specific simple version of (45) are given for the same boundary-value problem.

\section{INCREMENTAL EQUATIONS FOR THE COATED BLOCK}

\subsection{Incremental equations of motion}

The incremental equations of motion (16) are coupled with the constitutive law (17) to obtain a description in terms of displacement $\mathbf{u}(\mathbf{x}, t)$, which, in the polar coordinate system introduced in $\hat{\mathcal{C}}$, takes the form $\mathbf{u}=u_{r} \mathbf{e}_{r}+u_{\theta} \mathbf{e}_{\theta}$. With respect to the same basis the tensor $\Gamma$ is

$$
\boldsymbol{\Gamma}=u_{r, r} \mathbf{e}_{r} \otimes \mathbf{e}_{r}+\frac{u_{r, \theta}-u_{\theta}}{r} \mathbf{e}_{r} \otimes \mathbf{e}_{\theta}+u_{\theta, r} \mathbf{e}_{\theta} \otimes \mathbf{e}_{r}+\frac{u_{r}+u_{\theta, \theta}}{r} \mathbf{e}_{\theta} \otimes \mathbf{e}_{\theta}
$$

The incompressibility condition $(\operatorname{tr} \boldsymbol{\Gamma}=0)$ requires that

$$
r u_{r, r}+u_{r}+u_{\theta, \theta}=0
$$

For an incompressible isotropic elastic material the components of the constitutive fourthorder tensor $\mathbb{C}_{0}$ can be written in terms of two incremental moduli, denoted $\gamma_{1}$ and $\gamma_{2}$, that depend on the deformation. The non-vanishing components of $\mathbb{C}_{0}$ in (17) may be expressed in the form

$$
\begin{array}{ll}
C_{0 r r r r}=C_{0 \theta \theta \theta \theta}=2 \gamma_{1}+\tilde{p}, & C_{0 r \theta \theta r}=\gamma_{2}+\tilde{p}, \\
C_{0 r \theta r \theta}=\gamma_{2}-\frac{\sigma_{\theta}-\sigma_{r}}{2}, & C_{0 \theta r \theta r}=\gamma_{2}+\frac{\sigma_{\theta}-\sigma_{r}}{2},
\end{array}
$$


where $\tilde{p}=-\left(\sigma_{r}+\sigma_{\theta}\right) / 2$ and $\dot{p}$ in (17) is adjusted for consistency.

For hyperelastic materials, $\gamma_{1}$ and $\gamma_{2}$ are given in terms of the strain-energy function $\tilde{W}(\lambda)$ by

$$
\gamma_{1}=\frac{\lambda}{4}\left(\tilde{W}_{, \lambda}+\lambda \tilde{W}_{, \lambda \lambda}\right), \quad \gamma_{2}=\frac{\lambda}{2}\left(\frac{\lambda^{4}+1}{\lambda^{4}-1}\right) \tilde{W}_{, \lambda} .
$$

The incremental constitutive equations (17) can now be written as

$$
\begin{aligned}
\Sigma_{r r} & =\left(2 \gamma_{1}+\tilde{p}\right) u_{r, r}-\dot{p}, \\
\Sigma_{\theta \theta} & =\left(2 \gamma_{1}+\tilde{p}\right) \frac{u_{r}+u_{\theta, \theta}}{r}-\dot{p}, \\
\Sigma_{r \theta} & =\left(\gamma_{2}+\tilde{p}\right) \frac{u_{r, \theta}-u_{\theta}}{r}+\left(\gamma_{2}-\frac{\sigma_{\theta}-\sigma_{r}}{2}\right) u_{\theta, r}, \\
\Sigma_{\theta r} & =\left(\gamma_{2}+\frac{\sigma_{\theta}-\sigma_{r}}{2}\right) \frac{u_{r, \theta}-u_{\theta}}{r}+\left(\gamma_{2}+\tilde{p}\right) u_{\theta, r} .
\end{aligned}
$$

Since the underlying deformation is static, the incremental equations of motion, in polar coordinates, can be written as

$$
\begin{aligned}
& \Sigma_{r r, r}+\frac{\Sigma_{\theta r, \theta}}{r}+\frac{\Sigma_{r r}-\Sigma_{\theta \theta}}{r}=\rho u_{r, t t}, \\
& \Sigma_{r \theta, r}+\frac{\Sigma_{\theta \theta, \theta}}{r}+\frac{\Sigma_{r \theta}+\Sigma_{\theta r}}{r}=\rho u_{\theta, t t},
\end{aligned}
$$

where here and henceforth ${ }_{, t}$ denotes the material time derivative.

By substituting Equations (53) into (54) we obtain

$$
\begin{aligned}
\dot{p}_{, r}= & {\left[\left(2 \gamma_{1}+\tilde{p}\right)_{, r}+\frac{2\left(2 \gamma_{1}+\tilde{p}\right)}{r}\right] u_{r, r}+\left(2 \gamma_{1}+\tilde{p}\right) u_{r, r r}+\left(\gamma_{2}+\tilde{p}\right) \frac{u_{\theta, r \theta}}{r} } \\
& +\left(\gamma_{2}+\varsigma\right) \frac{u_{r, \theta \theta}-u_{\theta, \theta}}{r^{2}}-\rho u_{r, t t}, \\
\dot{p}_{, \theta}= & {\left[r\left(\gamma_{2}-\varsigma\right)_{, r}+\gamma_{2}-\varsigma\right]\left(u_{\theta, r}+\frac{u_{r, \theta}-u_{\theta}}{r}\right)+\left(\gamma_{2}-\varsigma\right) r u_{\theta, r r} } \\
& +\left(\gamma_{2}-2 \gamma_{1}\right) u_{r, r \theta}-\rho r u_{\theta, t t},
\end{aligned}
$$

where $\varsigma=\left(\sigma_{\theta}-\sigma_{r}\right) / 2$. In $(55)_{2}$ the connection (18) has been employed together with the equilibrium equation in terms of the principal Cauchy stresses, which takes the form $\sigma_{r, r}=\left(\sigma_{\theta}-\sigma_{r}\right) / r$.

The incompressibility condition (50) admits the introduction of a displacement function $\psi=\psi(r, \theta, t)$ such that 


$$
u_{r}=\frac{\psi_{, \theta}}{r}, \quad u_{\theta}=-\psi_{, r} .
$$

Substitution of (56) into (55) followed by elimination of $\dot{p}$ leads to a single incremental equation of motion in terms of $\psi$, namely

$$
\begin{aligned}
& C r^{4} \psi_{, r r r r}+2 D r^{2} \psi_{, r r \theta \theta}+E \psi_{, \theta \theta \theta \theta}+2\left(r C_{, r}+C\right) r^{3} \psi_{, r r r}+2\left(r D_{, r}-D\right) r \psi_{, r \theta \theta} \\
& +\left(r^{2} C_{, r r}+r C_{, r}-C\right)\left(r^{2} \psi_{, r r}-r \psi_{, r}\right)-\left(2 r D_{, r}-2 D+r^{2} C_{, r r}+r C_{, r}-C-E\right) \psi_{, \theta \theta} \\
& -\rho r^{2}\left(r^{2} \psi_{, r r t t}+r \psi_{, r t t}+\psi_{, \theta \theta t t}\right)=0 .
\end{aligned}
$$

In terms of the incremental moduli $\gamma_{1}$ and $\gamma_{2}$ and strain energy $\tilde{W}(\lambda)$, the parameters $C$, $D$ and $E$ introduced in (57) are given by

$$
\begin{aligned}
C & =\gamma_{2}-\frac{\sigma_{\theta}-\sigma_{r}}{2}=\frac{\lambda}{\lambda^{4}-1} \tilde{W}_{, \lambda}, \quad E=\gamma_{2}+\frac{\sigma_{\theta}-\sigma_{r}}{2}=\frac{\lambda^{5}}{\lambda^{4}-1} \tilde{W}_{, \lambda}, \\
D & =2 \gamma_{1}-\gamma_{2}=\frac{\lambda}{2}\left(\lambda \tilde{W}_{, \lambda \lambda}-\frac{2}{\lambda^{4}-1} \tilde{W}_{, \lambda}\right) .
\end{aligned}
$$

Equation (57) is strongly elliptic if $C>0, E>0$ and $D>-\sqrt{C E}$, or, in terms of $\tilde{W}$,

$$
\frac{\tilde{W}_{, \lambda}}{\lambda^{2}-1}>0, \quad \lambda \tilde{W}_{, \lambda \lambda}>-\frac{2 \tilde{W}_{, \lambda}}{\lambda^{2}+1} .
$$

\subsection{Boundary conditions}

Through (25), the incremental boundary conditions (21) can be written in component form as

$$
\begin{gathered}
-\Sigma_{r r}+\lambda^{-1} \rho_{0} w_{r, t t}=T, \quad-\Sigma_{r \theta}+\lambda^{-1} \rho_{0} w_{\theta, t t}=-N \text { on } \hat{P}^{-} \\
\Sigma_{r r}+\lambda^{-1} \rho_{0} w_{r, t t}=-T, \quad \Sigma_{r \theta}+\lambda^{-1} \rho_{0} w_{\theta, t t}=N \text { on } \hat{P}^{+} .
\end{gathered}
$$

The general expressions for $T$ and $N$, given in Equation (26), are now specialized for the problem under consideration. Since the underlying deformation is static, we have $\mathrm{D}^{2} \varphi / \mathrm{D} t^{2}=0$. Moreover, $\lambda$ and $\kappa$ are constant on $\hat{P}^{+}$and $\hat{P}^{-}$, so that $\lambda^{\prime}=\kappa^{\prime}=0$ and hence, from Equation (48), $F^{\prime}=M^{\prime}=0$.

Finally, from Equation (8), we have $\dot{F}=U_{, \lambda \lambda} \dot{\lambda}+U_{, \lambda \kappa} \dot{\kappa}$ and $\dot{M}=U_{, \lambda \kappa} \dot{\lambda}+U_{, \kappa \kappa} \dot{\kappa}$. Therefore, in (60) and (61) we use

$$
\begin{aligned}
T & =U_{, \lambda \lambda} \dot{\lambda}^{\prime}+U_{, \lambda \kappa} \dot{\kappa}^{\prime}-\lambda^{-1} \kappa\left(U_{, \lambda} \dot{\varphi}-U_{, \lambda \kappa} \dot{\lambda}^{\prime}-U_{, \kappa \kappa} \dot{\kappa}^{\prime}+\lambda^{-1} I \dot{\varphi}_{, t t}\right), \\
N & =\lambda^{-1} \dot{\kappa} U_{, \lambda}-U_{, \lambda \kappa} \dot{\lambda}^{\prime \prime}-U_{, \kappa \kappa} \dot{\kappa}^{\prime \prime}+\lambda^{-1} \kappa\left(U_{, \lambda \lambda} \dot{\lambda}+U_{, \lambda \kappa} \dot{\kappa}\right)+\lambda^{-1} I \dot{\varphi}_{, t t}^{\prime} .
\end{aligned}
$$


Equations $(60)_{1}$ and $(61)_{1}$ involve $\Sigma_{r r}$, which includes the unknown $\dot{p}$. This can be eliminated by differentiating the two conditions with respect to $\theta$ and substituting $(55)_{2}$ into $\Sigma_{r r, \theta}$. In terms of $\psi$ we then have

$$
\begin{aligned}
\Sigma_{r r, \theta}= & \left(2 D+C-\sigma_{r}\right) \frac{r \psi_{, r \theta \theta}-\psi_{, \theta \theta}}{r^{2}}+\left(r C_{, r}+C\right) \frac{r^{2} \psi_{, r r}-r \psi_{, r}-\psi_{, \theta \theta}}{r^{2}} \\
& +C r \psi_{, r r r}-\rho r \psi_{, r t t},
\end{aligned}
$$

and

$$
\Sigma_{r \theta}=\left(C-\sigma_{r}\right) \frac{r \psi_{, r}+\psi_{, \theta \theta}}{r^{2}}-C \psi_{, r r} .
$$

The final form of the boundary conditions on $\hat{P}$ for the incremental equation of motion (57) can be obtained through the specialization of (27), which yields

$$
\dot{\lambda}=-\lambda w_{r, r}, \quad \dot{\kappa}=\kappa \frac{w_{\theta, \theta}-w_{r, \theta \theta}}{r}, \quad \dot{\varphi}=\frac{w_{\theta}-w_{r, \theta}}{r},
$$

and noting that, from Equation (41), $\sigma_{r}=-\kappa \lambda^{-1} U_{, \lambda}$. Substitution in the expressions (62) for $T$ and $N$ and the use of ()$^{\prime}=\kappa \lambda^{-1}()_{, \theta}$ then yield

$$
\begin{aligned}
& U_{, \lambda \lambda} \lambda^{2}\left(\psi_{, \theta \theta}-r \psi_{, r \theta \theta}\right)-\lambda \kappa U_{, \lambda \kappa}\left(r \psi_{, r \theta \theta}+2 \psi_{, \theta \theta \theta \theta}-r \psi_{, r \theta \theta \theta \theta}\right) \\
+ & \kappa^{2} U_{, \kappa \kappa}\left(\psi_{, \theta \theta \theta \theta \theta \theta}+r \psi_{, r \theta \theta \theta \theta}\right)-\lambda U_{, \lambda}\left(\psi_{, \theta \theta \theta \theta}+\psi_{, \theta \theta}\right)-I\left(r \psi_{, r \theta \theta t t}+\psi_{, \theta \theta \theta \theta t t}\right) \\
= & \lambda^{2} \kappa^{-1}\left[\left(r C_{, r}+C\right)\left(\psi_{, \theta \theta}+r \psi_{, r}-r^{2} \psi_{, r r}\right)+(2 D+C)\left(\psi_{, \theta \theta}-r \psi_{, r \theta \theta}\right)\right. \\
- & \left.C r^{3} \psi_{, r r r}+\rho r^{3} \psi_{, r t t}\right] \pm \lambda \kappa^{-1} \rho_{0} r \psi_{, \theta \theta t t} \quad \text { on } \hat{P}^{\mp},
\end{aligned}
$$

and

$$
\begin{gathered}
\left(\lambda^{2} U_{, \lambda \lambda}+\lambda \kappa U_{, \lambda \kappa}\right)\left(\psi_{, \theta \theta}-r \psi_{, r \theta \theta}\right)-\left(\lambda \kappa U_{, \lambda \kappa}+\kappa^{2} U_{, \kappa \kappa}\right)\left(\psi_{, \theta \theta \theta \theta}+r \psi_{, r \theta \theta}\right) \\
\left.+\quad I\left(r \psi_{, r t t}+\psi_{, \theta \theta t t}\right)=\lambda^{2} \kappa^{-1} C\left(\psi_{, \theta \theta}+r \psi_{, r}-r^{2} \psi_{, r r}\right) \pm \lambda \kappa^{-1} \rho_{0} r^{2} \psi_{, r t t} \text { on } \hat{P}^{\mp} .(67)\right)
\end{gathered}
$$

For an uncoated block, boundary conditions on the two curved sides correspond to imposition of traction-free surface conditions, namely

$$
\begin{gathered}
C r^{3} \psi_{, r r r}-(2 D+C)\left(\psi_{, \theta \theta}-r \psi_{, r \theta \theta}\right)-r^{3} \rho \psi_{, r t t}=0 \quad \text { on } \hat{P}^{\mp}, \\
\psi_{, \theta \theta}+r \psi_{, r}-r^{2} \psi_{, r r}=0 \quad \text { on } \hat{P}^{\mp} .
\end{gathered}
$$

The moment of inertia $I$ (per unit length in $P$ ) can be calculated using the analogy with plate theory by considering the coating to be a rod of length $v$ (the deformed coating thickness). The moment of inertia about the axis $\mathbf{k}$ through its centre is then $I=\rho_{0} v^{2} / 12$. 
Let $\rho_{0}=\rho_{c} V$, where $\rho_{c}$ is the mass density of the film per unit reference area and $V$ is the film thickness in the reference configuration. Then

$$
I=\frac{\rho_{c} v^{2} V}{12}
$$

For simplicity, we assume that the coating material is incompressible. This leads to the equality $v=V / \lambda$ and hence

$$
I=\frac{\rho_{c} V^{3}}{12 \lambda^{2}}
$$

\section{DYNAMIC BEHAVIOUR}

Separable solutions of (57) of time-harmonic form are now examined. We represent the function $\psi(r, \theta, t)$ as

$$
\psi(r, \theta, t)=\phi(r) \cos q \theta \mathrm{e}^{\mathrm{i} \omega t}
$$

where $\phi(r)$ is a real function, $q$ is a real number to be determined and $\omega$ is the frequency of vibration.

Substitution of (72) into (73) yields a fourth-order linear ordinary differential equation for $\phi(r)$, not given explicitly here, which is solved numerically. To this end, it is transformed into a system of four first-order ordinary differential equations in the form

$$
z_{i}^{\prime}=\zeta_{i}\left(r, z_{1}, z_{2}, z_{3}, z_{4}\right), \quad i \in\{1,2,3,4\}
$$

where $z_{1}(r)=\phi(r), z_{2}(r)=\phi^{\prime}(r), z_{3}(r)=\phi^{\prime \prime}(r), z_{4}(r)=\phi^{\prime \prime \prime}(r)$. A general solution of (73) is given in terms of four independent solutions obtained by solving, using a standard Runge-Kutta method, four initial-value problems with initial conditions $z_{i}\left(r_{-}\right)=\delta_{i j}, i, j \in$ $\{1,2,3,4\}$, where $j$ is associated with the relevant initial-value problem and $r_{-}$is the value of $r$ on the boundary $P^{-}$. When the general solution is introduced into the boundary conditions (66) and (67) a homogeneous linear system of fourth order is obtained. This admits nontrivial solutions if the determinant of the associated matrix of coefficients vanishes, and this yields the 'frequency' equation.

As in [5], we impose the incremental conditions

$$
u_{r}=0, \quad \Sigma_{\theta \theta}=0
$$

on $\theta= \pm \alpha H$. The first condition requires that $\psi_{, \theta}=0$ on $\theta= \pm \alpha H$ and is satisfied provided $q$ is given by

$$
q=\frac{k \pi}{\alpha H}
$$


where $k$ is an integer (taken to be positive without loss of generality). With the choice (72) for $\psi(r, \theta, t), H$ and $k$ appear in Equation (57) and the boundary conditions (66) and (67) only through $q$. It therefore suffices to set $k=1$ in (75). Results for $k>1$ can be obtained from those for $k=1$ by the scaling $H / k$ of $H$.

As already pointed out in section 4.1, the bulk solid is modelled by a three-term strain energy of the form (45) and the coating behaviour is described by the strain energy (47). All parameters appearing in the formulation are non-dimensionalized using the shear modulus $\mu$ and the length scale $A$, and an overbar signifies a dimensionless quantity. The dimensionless parameters for the coating material are

$$
\bar{m}=\frac{m}{\mu A}, \quad \bar{n}=\frac{n}{\mu A^{3}} .
$$

The dimensionless form $\bar{\Omega}$ of the squared frequency is defined as

$$
\bar{\Omega}=\frac{\rho \omega^{2} A^{2}}{\mu}
$$

and the dimensionless form of the moment of inertia $I$, given by (71), is

$$
\bar{I}=\frac{\rho_{c} \bar{V}^{3}}{12 \rho \lambda^{2}}
$$

where $\bar{V}=V / A$.

As previously discussed, $V$ is proportional to $\sqrt{n / m}$. In particular, it can be shown that, in dimensionless form,

$$
\bar{V}=2 \sqrt{\frac{3 \bar{n}}{\bar{m}}} .
$$

In dimensionless form, the requirement $n / m \ll A^{2}$ becomes

$$
\bar{n} / \bar{m} \ll 1 .
$$

\subsection{Numerical results}

The dynamic behaviour of a coated block subject to bending is now illustrated with some numerical examples. For this purpose we concentrate on results where the thin film occupies only one boundary, $P^{-}$or $P^{+}$.

The response of the structure depends on the following parameters:

- the initial dimensions of the block through the ratio $H / A$;

- the extent of the bending deformation given by $\alpha$;

- the material properties of the block fixed by the constitutive assumptions described in section 4.1;

- the density of the coating relative to that of the bulk material;

- $\bar{m}$ and the ratio $\bar{n} / \bar{m}$. 
When the values of these parameters are given it remains to determine the dimensionless frequency $\bar{\Omega}$ from the frequency equation.

Numerical results for a coating on $P^{+}$are reported in Figures 1-3. In Figures 1 and 2, for $H / A=1,2$ respectively, the parameter $\bar{\Omega}$ is plotted against the stretch $\lambda_{+}$on the longer boundary. Illustrative plots are reported for $\bar{m}=1$ with $\bar{n} / \bar{m}=0.001,0.01,0.02$. Results for lower and higher values of $\bar{n}$ show little qualitative difference from those considered here. Variations in $\bar{m}$ have been explored but no significant differences have been found for $\bar{m}=0.1,0.01$. For convenience $A$ is set equal to 1 throughout.

The influence of the relative densities of the bulk and film materials is investigated by considering density ratios $\rho_{c} / \rho=0.1,1,10$. In general, continuous curves pertain to the coated block, while dashed curves describe the behaviour of the uncoated block. Several solution branches are shown. Their cut-off point on the right of the figures corresponds to the value of $\lambda_{+}$for which the plane section of the block is deformed into a circular annulus, at which point $\alpha H=\pi$. In particular, with $\bar{m}=1$, the limiting values are $\lambda_{+} \approx 3.55,2.53$ for $H / A=1,2$, respectively.

The first branch in each plot intersects the axis $\bar{\Omega}=0$ at a point coinciding with the critical value of $\lambda_{+}$corresponding to bifurcation. Dryburgh and Ogden [5] have found that a coating on $P^{+}$promotes bifurcation and that the value of the critical stretch at bifurcation, denoted $\lambda_{+}^{\text {bif }}$, does not depend significantly on the flexural stiffness. For $H / A=1,2$, $\lambda_{+}^{\text {bif }} \approx 1.66,1.84$, respectively, which can be compared to the corresponding values $\lambda_{+}^{\text {bif }} \approx$ $1.88,2.16$ for an uncoated block. Solution branches beyond a critical value of $\lambda_{+}$cannot be reached in a stable quasi-static deformation starting from the undeformed rectangular configuration.

Figure 1 is for $H / A=1$. The first row of plots shows that, when the density of the bulk material dominates that of the coating, an increase of bending stiffness has no effect on the frequency of vibration. In the vicinity of the undeformed configuration $\left(\lambda_{+}=1\right)$ the frequency for the coated block is close to that in the uncoated case, especially for the lower solution branches. In general, the coating has the effect of lowering the frequencies of the first branch and, as indicated above, bifurcation occurs earlier than for the uncoated block. When the densities of the substrate and the film are equal $\left(\rho_{c} / \rho=1\right)$, an increase in the bending stiffness influences primarily the upper branches, whereas the lower branch frequencies are only slightly reduced. When the film density is greater the values of $\bar{\Omega}$ for the first three branches decrease significantly as $\bar{n} / \bar{m}$ increases while the other solution branches are virtually unaffected. Figure 2 shows the results for $H / A=2$ for $\rho_{c} / \rho=1,10$. Apart from a necessary re-scaling of the abscissa axis, the plots are quite similar to those of Figure 1.

The effect of varying the mode parameter $q=k \pi /(\alpha H)$ on $\bar{\Omega}$ at $\lambda_{+}=1.3$ is shown in Figure 3. By means of $q$ different modes may be selected through the ratio $H / k$. Moreover, it allows for varying the 'fan' described by the flexed block through $\alpha H$ (cf Equation (31)). Since the block deforms into an annulus when $\alpha H=\pi$, the $q$-axis starts from 1 . Instead of $\bar{\Omega}$, the variable plotted on the vertical axis is $\bar{\Omega} / q^{2}$, which is a dimensionless measure of the square of the speed of propagation of a wave along a curved boundary. Since the wavelength is $\Lambda=2 H / k=2 \pi /(\alpha q)$ the frequency $\omega$, which, by definition, is $\omega=2 \pi c / \Lambda$, can be written as $\omega=\alpha q c$, where $c$ is the wave speed. It turns out that

$$
\frac{\bar{\Omega}}{q^{2}}=\frac{\rho \alpha^{2} A^{2}}{\mu} c^{2} .
$$



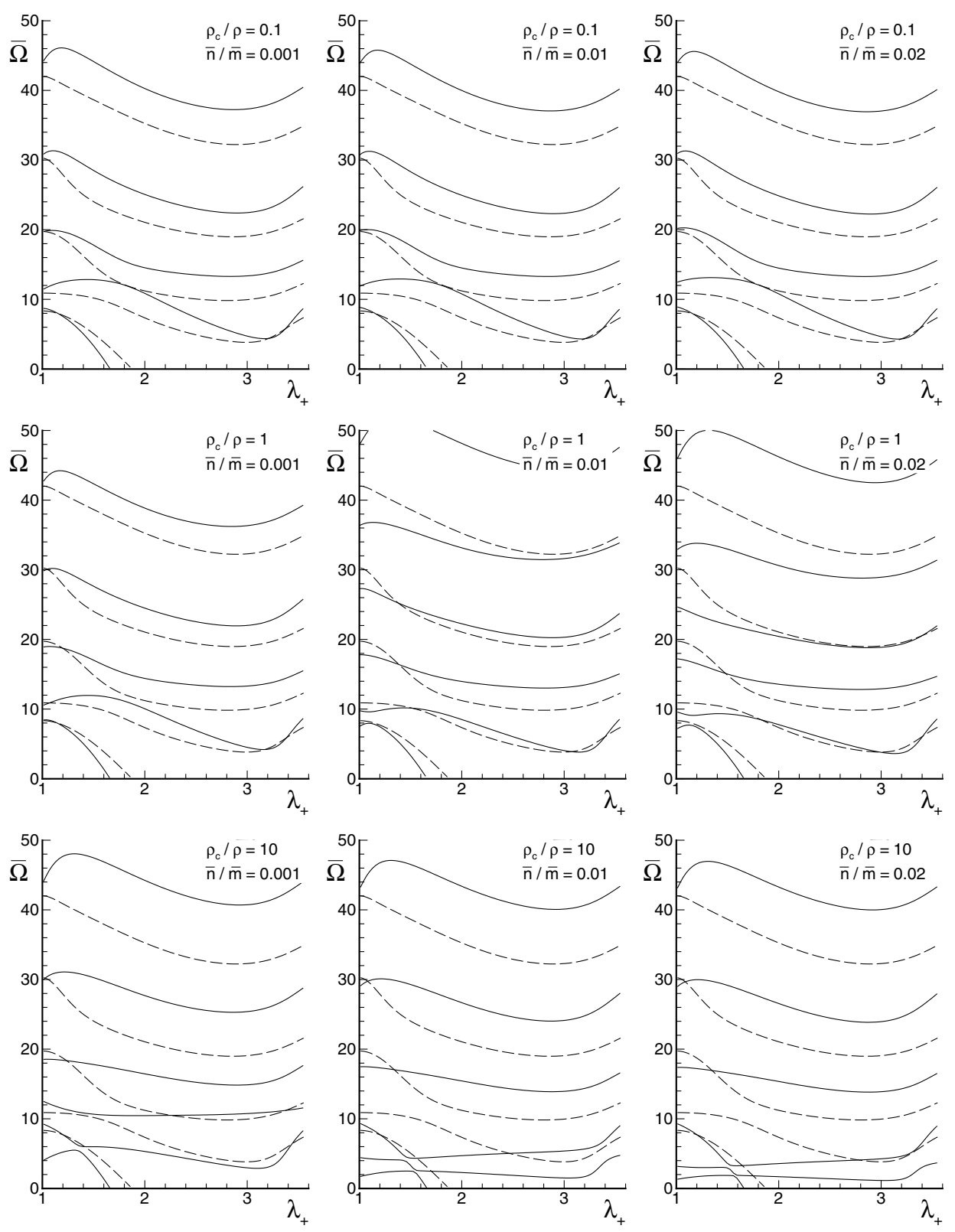

Figure 1. Plots of $\bar{\Omega}$ against $\lambda_{+}$for an Ogden material block coated on $P^{+}$(solid curves) with $H / A=1$, $\bar{m}=1$; dashed curves are for an uncoated block. 

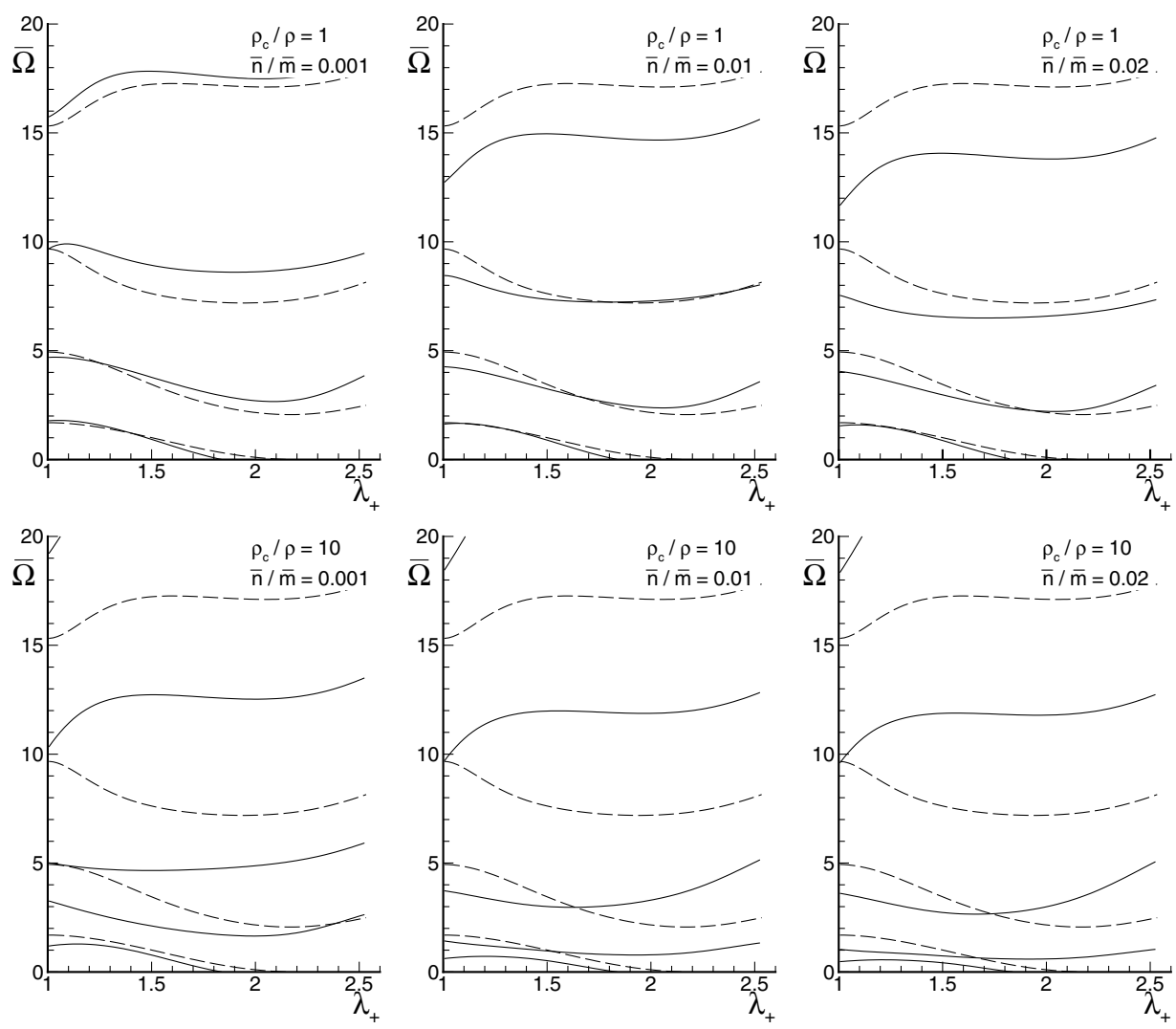

Figure 2. Plots of $\bar{\Omega}$ against $\lambda_{+}$for an Ogden material block coated on $P^{+}$(solid curves) with $H / A=2$, $\bar{m}=1$; dashed curves are for an uncoated block.

The first branch in each plot is affected by the pathological situation that $\bar{\Omega}=0$ when $q=1$, corresponding to an apparent bifurcation. A straightforward calculation shows that, for $k=1$ and $\lambda_{+}=1.3, q=1$ corresponds to $H / A=11.44,10.82$ for the uncoated and coated blocks, respectively. But from the results given in [5] it is evident that no bifurcation occurs for specimens of these dimensions. In fact, for $q=1$ the equations can be solved exactly and the resulting solution corresponds to a rigid rotation, which does not qualify as an acceptable bifurcation solution. The point $\left(\bar{\Omega} / q^{2}, q\right)=(0,1)$ is therefore associated with the trivial solution. Of course, in the limit $q=1$ the boundary conditions (74) are not applicable. The general trend noticed in Figures 1 and 2 is also apparent in Figure 3. In particular, the behaviour of the block is sensitive to changes in bending stiffness only if the coating density is greater than that of the block material.

Figures 4 and 5 are for a coating on $P^{-}$. Figure 4 results for a block with $H / A=1$, and density ratios $\rho_{c} / \rho=1,10$ are given. In contrast to the case when the coating is on $P^{+}$, bifurcation is advanced or delayed depending primarily on the ratio $\bar{n} / \bar{m}$. In this case the 

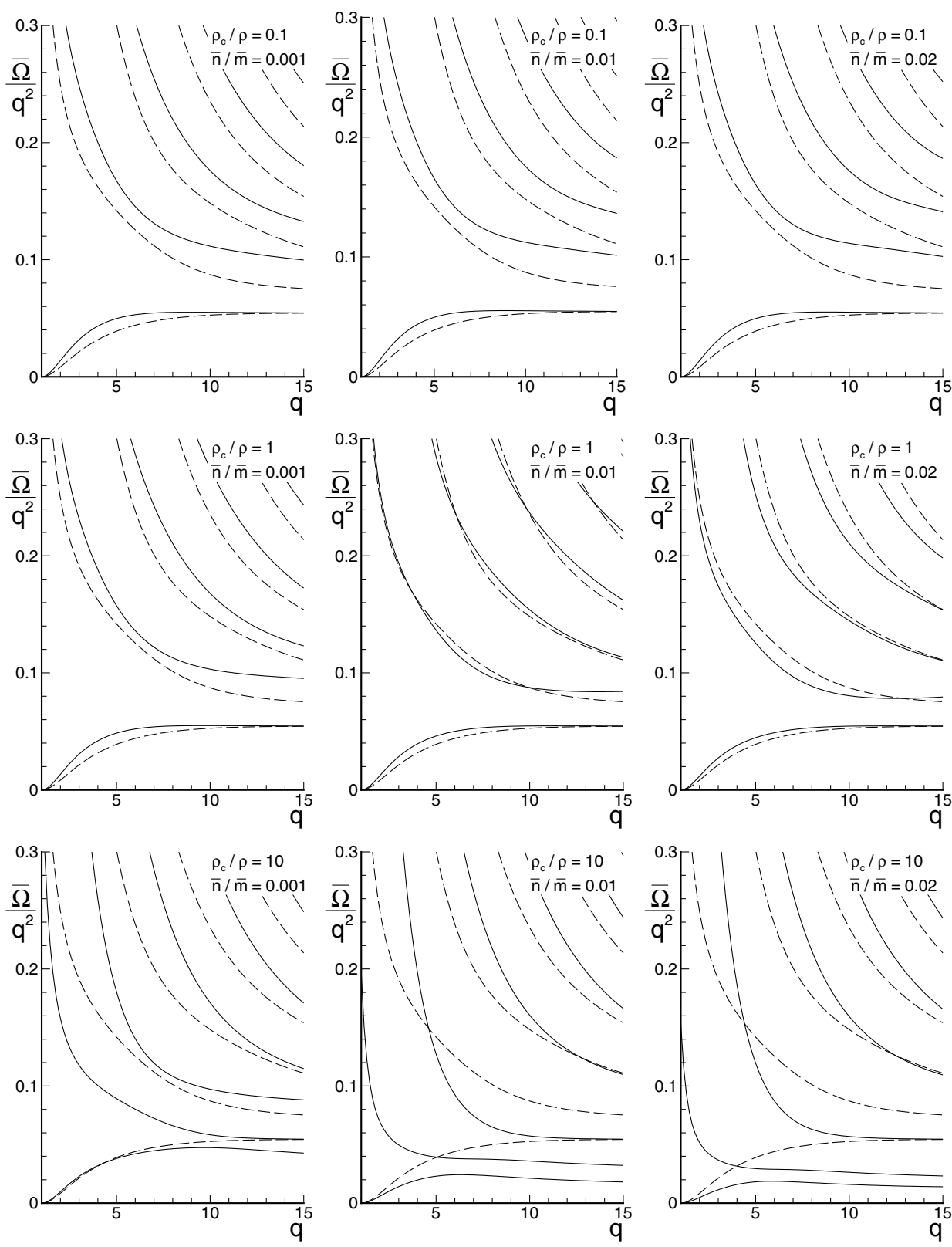

Figure 3. Plots of $\bar{\Omega} / q^{2}$ against $q$ for an Ogden material block coated on $P^{+}$(solid curves) at $\lambda_{+}=1.3$, $\bar{m}=1$; dashed curves are for an uncoated block. 

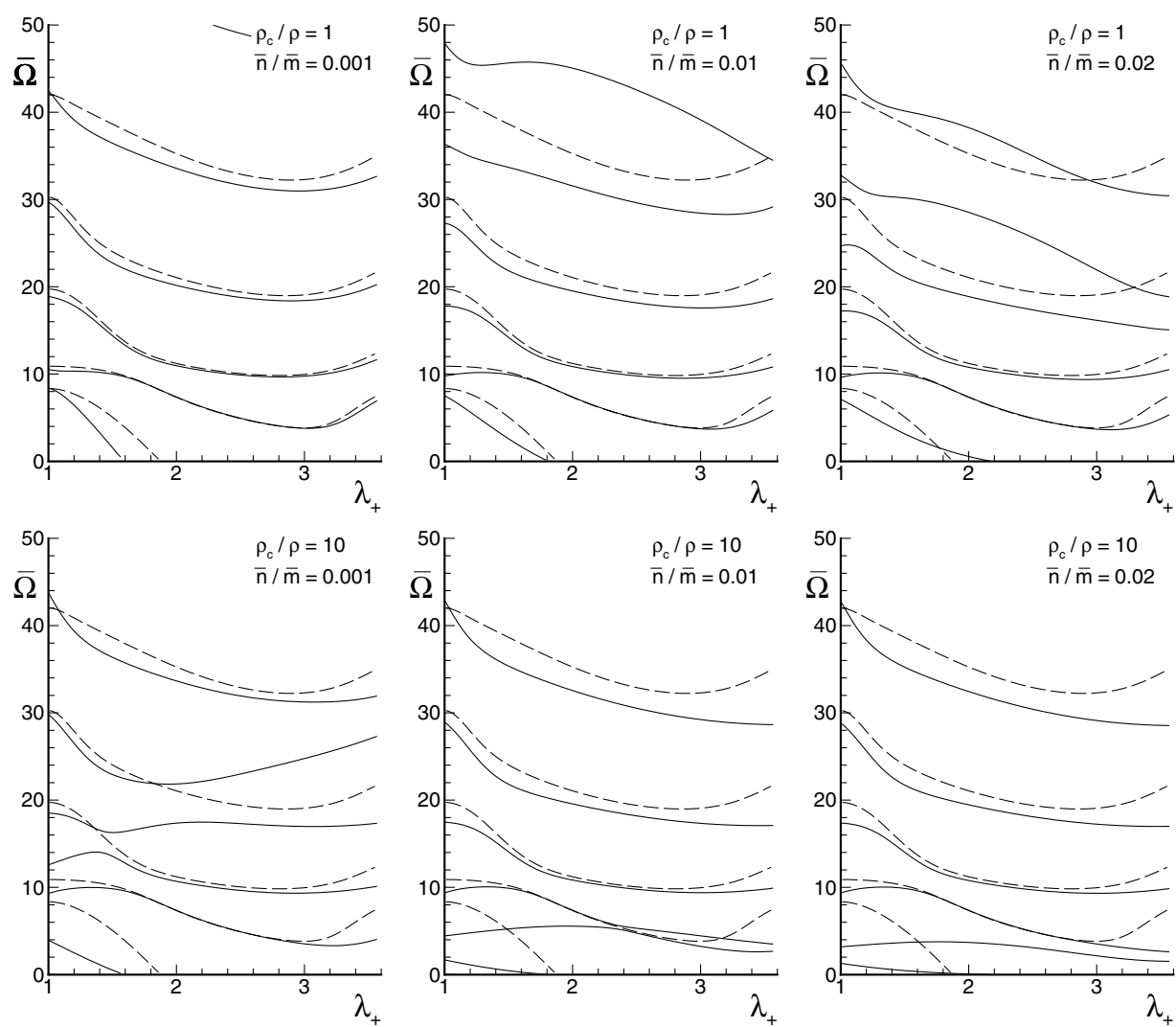

Figure 4. Plots of $\bar{\Omega}$ against $\lambda_{+}$for an Ogden material block coated on $P^{-}$(solid curves) with $H / A=1$, $\bar{m}=1$; dashed curves are for an uncoated block.

critical stretch $\lambda_{+}^{\text {bif }}$ lies in the range $[1.59,2.17]$ for both values of the density ratio. Also, when the coating is on $P^{-}$changes in $\bar{m}$ do not alter significantly the general behaviour, as found for a coating on $P^{+}$.

Figure 5 is the counterpart of Figure 3 for $\lambda_{+}=1.3$. Compared to the latter, the most apparent difference is that, as the mode wavelength decreases, a coating on the compressed side appreciably lowers the frequencies of vibration when the bending stiffness is small $(\bar{n} / \bar{m}=0.001)$ and for all density ratios. This can be interpreted as an indication of the tendency of the compressed film to be unstable when its behaviour is close to that of a membrane.

In Figure 6, the influence of the coating rotatory inertia term on the frequencies of vibration of an undeformed block is considered. To this end, the boundary conditions (21) and (22) are specialized for the case in which the block remains rectangular with $\lambda=1$. On $P^{+}$we then have

$$
\boldsymbol{\tau}=\mathbf{e}_{2}, \quad \boldsymbol{\nu}=-\mathbf{e}_{1}, \quad \kappa=0, \quad M^{\prime}=0, \quad G=0 .
$$



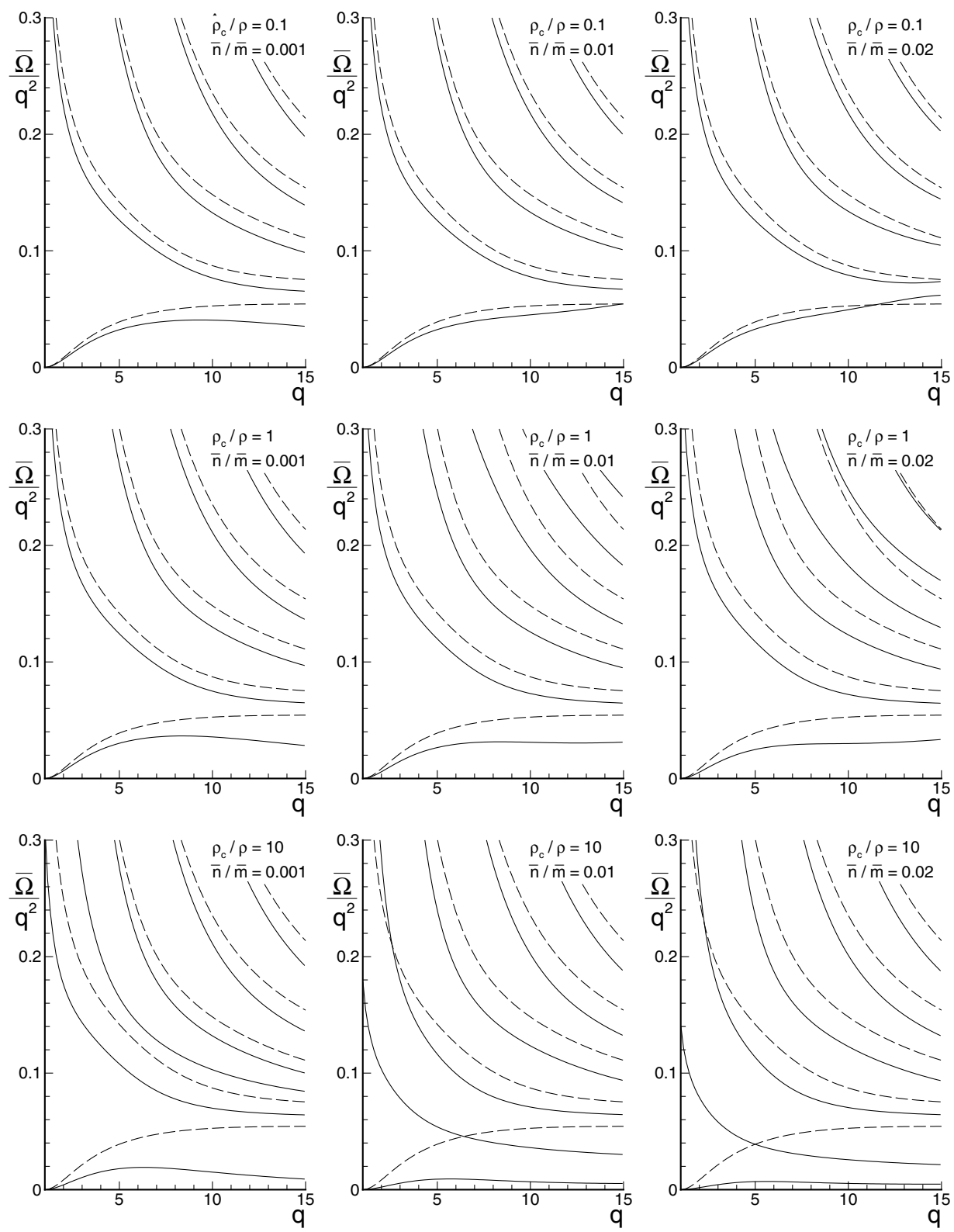

Figure 5. Plots of $\bar{\Omega} / q^{2}$ against $q$ for an Ogden material block coated on $P^{-}$(solid curves) at $\lambda_{+}=1.3$, $\bar{m}=1$; dashed curves are for an uncoated block. 

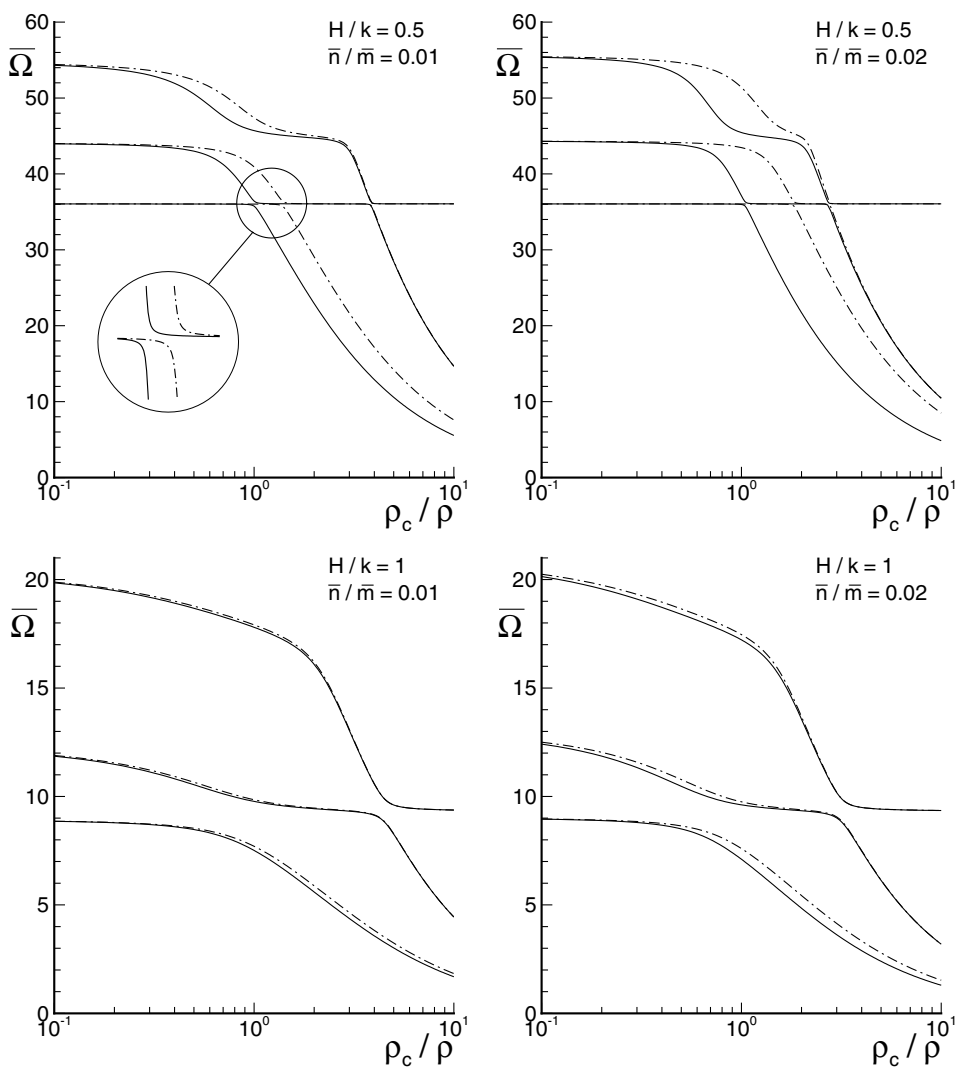

Figure 6. Plots of $\bar{\Omega}$ against $\rho_{c} / \rho$ for an elastic, undeformed block $\left(\lambda_{+}=\lambda_{-}=1\right)$ (solid curves), $\bar{m}=1$; dash-dotted curves are for $\bar{I}=0$.

As expected, the differences are more pronounced when the wavenumber (inversely proportional to $H / k$ ) is relatively high, but are essentially independent of the bending stiffness of the coating. In each plot, the first three solution branches are shown. For $H / k=0.5$ and for the first branch, the discrepancy between results for $\bar{I}=0$ and $\bar{I} \neq 0$ increases up to $100 \%$ when $\rho_{c} / \rho=10$. For $H / k>1$ the results are indifferent to inclusion of the coating inertia.

Acknowledgments. The authors acknowledge the PhD Thesis of Dr Gillian Dryburgh, which is related to the current work. M. G. is grateful for support from the University of Trento, Italy.

\section{REFERENCES}

[1] Steigmann, D. J. and Ogden, R. W.: Plane deformations of elastic solids with intrinsic boundary elasticity. Proc. R. Soc. Lond., A 453, 853-877 (1997).

[2] Steigmann, D. J. and Ogden, R. W.: A necessary condition for energy-minimizing plane deformations of elastic solids with intrinsic boundary elasticity. Math. Mech. Solids, 2, 3-16 (1997).

[3] Steigmann, D. J. and Ogden, R. W.: Elastic surface-substrate interactions. Proc. R. Soc. Lond., A 455, 437-474 (1999). 
[4] Ogden, R. W., Steigmann, D. J. and Haughton, D. M.: The effect of elastic surface coating on the finite deformation and bifurcation of a pressurized circular annulus. J. Elasticity, 47, 121-145 (1997).

[5] Dryburgh, G. and Ogden, R. W.: Bifurcation of an elastic surface-coated incompressible isotropic elastic block subject to bending. ZAMP, 50, 822-838 (1999).

[6] Ogden, R. W. and Steigmann, D. J.: Plane strain dynamics of elastic solids with intrinsic boundary elasticity, with application to surface wave propagation. J. Mech. Phys. Solids, 50, 1869-1896 (2002).

[7] Ogden, R. W.: Non-linear Elastic Deformations, Ellis Horwood, Chichester, 1984.

[8] Ogden, R. W.: Large deformation isotropic elasticity - on the correlation of theory and experiment for incompressible rubberlike solids. Proc. R. Soc. Lond., A 326, 565-584 (1972). 